\title{
Linear Dispersion Code with an Orthogonal Row Structure for Simplifying Sphere Decoding
}

\author{
X. G. Dai, S. W. Cheung and T. I. Yuk \\ Department of Electrical \& Electronic Engineering \\ The University of Hong Kong \\ Hong Kong \\ xgdai@eee.hku.hk, swcheung@eee.hku.hk, tiyuk@eee.hku.hk
}

\begin{abstract}
In this paper, we propose a family of full diversity fast decodable Linear Dispersion Codes (LDCs) for use in MIMO systems. These codes have as many as possible the orthogonal rows in the dispersion matrices of the LDC. One of the main advantages of this family of LDCs is that it can be used in MIMO systems with arbitrary numbers of transmitted and received antennas and arbitrary transmission rates. We also develop a simplified Sphere Decoding (SD) algorithm to significantly reduce the decoding complexity for this family of LDCs. Monte Carlo simulation shows that the optimum LDCs with or without this orthogonal structure have nearly identical bit-error rate (BER) performances, but the complexity can be significantly reduced. For a $2 \times 4$ MIMO system transmitting 8 QPSK symbols in a block length of 4 , the reduction is about $28-52 \%$, and for a $3 \times 3$ MIMO system transmitting 10 QPSK symbols in a block length of 6 , the reduction is about $28-49 \%$.
\end{abstract}

\section{Keywords- MIMO, Sphere decoding, orthogonal, complexity}

\section{INTRODUCTION}

Linear Dispersion Code (LDC) [1] is well-known for its advantage in providing full-ergodic capacity and good error probability performance to Multiple-Input Multiple-Output (MIMO) communication systems. However, to achieve the optimum bit-error rate (BER) performance, the tremendous complexity of the optimum Maximum Likelihood (ML) decoding process makes the implementation of high speed LDCs impractical. Minimum-Mean-Square Error (MMSE) [2] and Zero-Forcing (ZF) [3] algorithms are much less complicated, but the achievable BER performances are not very satisfactory.

Researchers have been working hard to develop simple decoding algorithms to achieve optimum BERs for LDCs. Alamouti [4] proposed a remarkable space-time (ST) code in 1998 for MIMO systems with two transmit antennas. Later on in [5], orthogonal space-time block codes (OSTBCs) were proposed with the same properties as Alamouti's code, i.e. allowing the use of a very simple ML decoding algorithm. However, the main disadvantage of the OSTBCs is that they cannot achieve full-transmission rates for MIMO systems with more than two transmit antennas [6]. Quasi-orthogonal STBCs [7] were then proposed to support full-transmission rates, but at a cost of lower diversity gains.

In [8], Sphere Decoding (SD) was proposed to substantially reduce the complexity of ML decoding, yet having the same
BER performance. SD can be applied to all linear STBCs. However, as Jalden et al. pointed out [9], for a fixed signal-tonoise ratio (SNR), the complexity of SD increases exponentially with the number of symbols jointly decoded and this makes SD still too complicated for high-data-rate transmissions.

Improvements in different aspects of SD have been proposed. In [10], a so-called Babai Point method was used to set the initial searched point in SD. In [11], a Schnorr Euchner (SE) enumeration method was proposed to refine the search strategy of SD. In [12], Paredes et al. reduced the complexity of SD by reducing the number of searched levels in the tree search process. By combining the advantages of OSTBCs and $\mathrm{SD}$, they constructed a family of fast decodable full-rate, full diversity codes for a $2 \times 2$ MIMO system. Biglieri et al. extended this concept to a $4 \times 2$ MIMO system and developed a family of quasi-orthogonal structured codes, leading to a reduction of 4 levels in the tree search for SD [13]. A new family of fast-decodable, full-rank, flexible-rate linear dispersion codes (LDCs) for MIMO systems with arbitrary numbers of transmit and receive antennas was proposed in [14]. This new family of LDCs has as many as possible the orthogonal rows in the dispersion matrices. The advantages of the orthogonal structure for different modulations were studied in [15]. In this paper, we study this family of LDCs for different numbers of transmit and receive antennas. We also use a simplified SD for the codes to achieve the same BER performance as conventional SD or ML decoding, but with much less decoding complexity.

The rest of this paper is organized as follows. The system model used for the study is defined in Section 2. The principle of SD is described in Section 3. In Section 4, the family of LDCs together with the corresponding simplified SD algorithm is described. Monte Carlo simulation results and discussions are given in Section 5. Section 6 concludes this paper.

\section{SySTEM MODEL}

The system model used for the study is an $N_{t} \times N_{r}$ MIMO system with $N_{t}$ transmit antennas and $N_{r}$ receive antennas, over a quasi-static Rayleigh fading channel. The $N_{r} \times T$ received signal matrix $\mathbf{R}$ is given by:

$$
\mathbf{R}=\mathbf{H C}+\mathbf{W}
$$


where the entries of $\mathbf{H} \in \mathbb{C}^{N_{r} \times N_{t}}$ represent the channel coefficients which are assumed to be perfectly known at the receiver but not at the transmitter, $\mathbf{C} \in \mathbb{C}^{N_{t} \times T}$ is the codeword matrix with block length $T$, and $\mathbf{W} \in \mathbb{C}^{N_{r} \times T}$ represents the complex additive white Gaussian noise (AWGN) matrix with elements being independently and identically distributed (iid) and following the normal distribution $N_{\mathrm{C}}\left(0, N_{0}\right)$.

For LDCs, the codeword $\mathbf{C}$ can be expressed as [1]:

$$
\mathbf{C}=\sum_{i=1}^{N} \mathbf{M}_{i} \cdot s_{i}
$$

where $\left\{\mathbf{M}_{i}\right\}_{i=1}^{N}$ are the dispersion matrices of the LDC, $\left\{s_{i}\right\}_{i=1}^{N}$ are the transmitted symbols taking values from some complex constellation in a finite set $\mathbb{S}$, and $N$ is the number of symbols in one codeword. All elements in $\mathbf{R}, \mathbf{H}, \mathbf{C}$ and $\mathbf{W}$ of (1) are complex variables. The transmitted symbols in (1) can be expressed in vector form as $\mathbf{s}=\left[s_{1}, s_{2}, s_{3}, s_{4} \ldots, s_{N}\right]$. Then substituting (2) into (1) and taking vectorization on both sides yields [1]

$$
\overline{\mathbf{r}}=\mathbf{K} \overline{\mathbf{X}} \overline{\mathbf{s}}+\overline{\mathbf{w}}
$$

where $\quad \overline{\mathbf{r}}=\operatorname{vec}(\mathbf{R})=\left[r_{1,1}, r_{2,1}, \ldots, r_{N_{r}, 1}, r_{1,2}, \ldots, r_{N_{r}, 2}, \ldots, r_{N_{r}, N_{t}}\right]^{T}$ with $r_{i, j}$ being the entry in the $i^{\text {th }}$ row and $j^{\text {th }}$ column of matrix $\mathbf{R}$ and []$^{T} \quad$ denoting matrix transposition, $\overline{\mathbf{X}}=\left[\operatorname{vec}\left(\mathbf{M}_{0}\right), \operatorname{vec}\left(\mathbf{M}_{1}\right), \ldots, \operatorname{vec}\left(\mathbf{M}_{N}\right)\right] \quad, \quad \overline{\mathbf{s}}=\operatorname{vec}(\mathbf{s})=\mathbf{s}^{T}$ $=\left[s_{1}, \ldots s_{N}\right]^{T}$ and $\overline{\mathbf{w}}=\operatorname{vec}(\mathbf{W})$.

In (3), $\mathbf{K}=\mathbf{I} \otimes \mathbf{H}$, where $\mathbf{K} \in \mathbb{C}^{N_{r} T \times N_{t} T}, \mathbf{I}$ is an identity matrix and $\otimes$ denotes the Kronecker product.

\section{SPHERE DECODING}

To facilitate the implementation of SD decoding, we need to transform the model using complex variables in (3) to an equivalent model using real variables as follows. Separating the real and imaginary parts of the elements in $\overline{\mathbf{r}}, \overline{\mathbf{s}}$ and $\overline{\mathbf{w}}$ of (3) and then taking vectorization on both sides give the realvalued expression:

$$
\tilde{\mathbf{r}}=\tilde{\mathbf{G}} \tilde{\mathbf{s}}+\tilde{\mathbf{W}}
$$

$$
\text { where } \quad \tilde{\mathbf{r}}=\left[\operatorname{Re}\left(\overline{\mathbf{r}}^{T}\right), \operatorname{Im}\left(\overline{\mathbf{r}}^{T}\right)\right]^{T} \quad, \quad \tilde{\mathbf{s}}=[\operatorname{Re}(\mathbf{s}), \operatorname{Im}(\mathbf{s})]^{T}
$$$$
=\left[\operatorname{Re}\left(s_{1}\right), \ldots, \operatorname{Re}\left(s_{N}\right), \operatorname{Im}\left(s_{1}\right), \ldots, \operatorname{Im}\left(s_{N}\right)\right]^{T}
$$
$\tilde{\mathbf{w}}=\left[\operatorname{Re}\left(\overline{\mathbf{w}}^{T}\right), \operatorname{Im}\left(\overline{\mathbf{w}}^{T}\right)\right]^{T}$, and $\operatorname{Re}($.$) and \operatorname{Im}($.$) denote the real$ and imaginary parts of (.), respectively. In (4), $\tilde{\mathbf{G}}$ is a $2 N_{r} T \times 2 N$ real matrix given by:

$$
\tilde{\mathbf{G}}=\left[\begin{array}{cc}
\operatorname{Re}(\mathbf{K} \overline{\mathbf{X}}) & -\operatorname{Im}(\mathbf{K} \overline{\mathbf{X}}) \\
\operatorname{Im}(\mathbf{K} \overline{\mathbf{X}}) & \operatorname{Re}(\mathbf{K} \overline{\mathbf{X}})
\end{array}\right]
$$

Here we arrange the vector $\tilde{\mathbf{s}}$ to

$$
\tilde{\tilde{\mathbf{s}}}=\left[\operatorname{Re}\left(s_{1}\right), \operatorname{Im}\left(s_{1}\right), \operatorname{Re}\left(s_{2}\right), \operatorname{Im}\left(s_{2}\right), \ldots, \operatorname{Re}\left(s_{N}\right), \operatorname{Im}\left(s_{N}\right)\right]^{T}
$$

$$
=\left[\tilde{\tilde{s}}_{1}, \tilde{\tilde{s}}_{2}, \tilde{\tilde{s}}_{3}, \tilde{\tilde{s}}_{4} \ldots, \tilde{\tilde{s}}_{2 N}\right]^{T}
$$

To keep the received signal vector $\tilde{\mathbf{r}}$ in (4) unchanged, we need to arrange the columns of $\tilde{\mathbf{G}}$ correspondingly. We denote the columns of $\tilde{\mathbf{G}}$ as $\left[\operatorname{col}_{1}(\tilde{\mathbf{G}}), \operatorname{col}_{2}(\tilde{\mathbf{G}}), \ldots, \operatorname{col}_{2 N}(\tilde{\mathbf{G}})\right]$, where $\operatorname{col}_{i}(\tilde{\mathbf{G}})$, for $i=1,2, \ldots, 2 N$, is the $i^{\text {th }}$ column of $\tilde{\mathbf{G}}$, and we re-arrange the columns of $\tilde{\mathbf{G}}$ to give:

$$
\tilde{\tilde{\mathbf{G}}}=\left[\operatorname{col}_{1}(\tilde{\mathbf{G}}), \operatorname{col}_{N+1}(\tilde{\mathbf{G}}), \operatorname{col}_{2}(\tilde{\mathbf{G}}), \ldots, \operatorname{col}_{N}(\tilde{\mathbf{G}}), \operatorname{col}_{2 N}(\tilde{\mathbf{G}})\right]
$$

With these arrangements, the received signal vector $\tilde{\mathbf{r}}$ in (4) can be re-written as:

$$
\tilde{\mathbf{r}}=\tilde{\tilde{\mathbf{G}}} \tilde{\tilde{\mathbf{S}}}+\tilde{\mathbf{w}}
$$
[8]

SD attempts to find the solution to the least square problem

$$
\hat{\mathbf{s}}=\arg \min _{\tilde{\tilde{\mathbf{s}}} \in \mathbb{S}}\|\tilde{\mathbf{r}}-\tilde{\tilde{\mathbf{G}}} \tilde{\tilde{\mathbf{s}}}\|^{2}
$$

Using the QR decomposition on matrix $\tilde{\tilde{\mathbf{G}}}$ gives

$$
\tilde{\tilde{\mathbf{G}}}=\mathbf{Q}\left[\begin{array}{c}
\mathbf{P} \\
\mathbf{0}_{\left(2 N_{r} T-2 N\right) \times 2 N}
\end{array}\right]
$$

where $\mathbf{P} \in \mathbb{R}^{2 N \times 2 N}$ is an upper triangular matrix, $\mathbf{Q}=\left[\mathbf{Q}_{1}\right.$, $\left.\mathbf{Q}_{2}\right] \in \mathbb{R}^{2 N_{r} T \times 2 N_{r} T}$ is an orthonormal matrix, and here $N_{r} T \geq N$ needs to be satisfied. Matrices $\mathbf{Q}_{1}$ and $\mathbf{Q}_{2}$ are the first $2 N$ and last $2 N_{r} T-2 N$ orthonormal columns of $\mathbf{Q}$, respectively. Due to the invariance of the Frobenius norm to orthogonal transforms, (9) can be re-written as [9]

$$
\hat{\mathbf{s}}=\arg \min _{\tilde{\mathbf{s}} \in \mathbb{S}}\|\mathbf{y}-\mathbf{P} \tilde{\tilde{\mathbf{s}}}\|^{2}
$$

where $\mathbf{y}=\mathbf{Q}_{1}^{T} \tilde{\mathbf{r}}$. SD searches for $\hat{\mathbf{s}}$ only over those points that satisfy the constraint

$$
\|\mathbf{y}-\mathbf{P} \tilde{\tilde{\mathbf{s}}}\|^{2} \leq d^{2}
$$

Denoting $\mathbf{a}=\mathbf{a}(\tilde{\mathbf{s}})=\mathbf{y}-\mathbf{P} \tilde{\tilde{\mathbf{s}}}$ as a function of $\tilde{\tilde{\mathbf{s}}},(12)$ can be written as

$$
\sum_{i=1}^{2 N} a_{i}^{2} \leq d^{2}
$$

where $a_{i}$ is the $i^{\text {th }}$ element of $\mathbf{a}$. Because of the upper triangular structure of $\mathbf{P}, a_{i}$ is a function of only $\tilde{\tilde{s}}_{2 N}, \tilde{\tilde{s}}_{2 N-1}, \ldots$, $\tilde{\tilde{s}}_{i}$, where $\tilde{\tilde{s}}_{j}$ denotes the $j^{\text {th }}$ element of $\tilde{\tilde{\mathbf{s}}}$. To begin the search, we first consider the inequality

$$
a_{2 N}^{2}\left(\tilde{\tilde{s}}_{2 N}\right) \leq d^{2}
$$

where $a_{2 N}\left(\tilde{\tilde{s}}_{2 N}\right)$ indicates that $a_{2 N}$ is a function of $\tilde{\tilde{s}}_{2 N} \cdot(14)$ is a necessary condition of (13) because $a_{i}^{2}$ is non-negative. Solving this inequality gives the upper and lower bounds of the 
possible values of $\tilde{\tilde{s}}_{2 N}$. For each choice of $\tilde{\tilde{s}}_{2 N}$, the following inequality

$$
a_{2 N-1}\left(\tilde{\tilde{s}}_{2 N-1}, \tilde{\tilde{s}}_{2 N}\right)^{2}+a_{2 N}\left(\tilde{\tilde{s}}_{2 N}\right)^{2} \leq d^{2}
$$

again a necessary condition of (13), gives the upper and lower bounds of the possible values of $\tilde{\tilde{s}}_{2 N-1}$. Going on in this way, the possible values of all the elements of $\tilde{\tilde{\mathbf{s}}}$ can be found. Here we denote (14) the $2 N^{\text {th }}$ level inequality, (15) the $(2 N-1)^{\text {th }}$ level and so on. During the searching process, when an inequality cannot be satisfied, the algorithm goes back to the previous level, selects another possible value of $\tilde{\tilde{s}}$ and then continues. After a possible vector $\tilde{\tilde{\mathbf{s}}}$ is found, the value of $d^{2}$ is reduced and the search starts all over again. The algorithm stops when (13) is violated for all possible values of $\tilde{\tilde{\mathbf{s}}}$, and the last found $\tilde{\tilde{\mathbf{s}}}$ is taken as the result.

\section{NEW LDC AND DeCoding Algorithm}

From the description in Section 3, we can see that the process of SD can be considered as a tree search [9]. In this section, we illustrate how we can reduce the number of levels in the tree search by using LDC with orthogonal row structure, hence to reduce the complexity of the SD [14].

\section{A. Orthogonal row structure}

This family of LDCs has the first $m$ dispersion matrices among the $N$ dispersion matrices $\left\{\mathbf{M}_{i}\right\}_{i=1}^{N}$ in (2) satisfying the following condition:

$$
<\mathbf{M}_{i}(p,:), \mathbf{M}_{j}(q,:)>=0
$$

for $i \neq j ; i, j \leq m ; 1 \leq p \leq N_{t}, 1 \leq q \leq N_{t}$

where $\mathbf{M}_{i}(k,:)$ denotes the $k^{\text {th }}$-row vector of $\mathbf{M}_{i}$ and $<\mathbf{a}, \mathbf{b}>$ denotes the inner product of vectors $\mathbf{a}$ and $\mathbf{b}$. This means that among the first $m$ dispersion matrices, the rows of one dispersion matrix are orthogonal to the rows of any other dispersion matrix. It can be easily proved that LDCs with the dispersion matrices satisfying (16) will satisfy the full-capacity constraint [1]. It can also be rigorously shown that for the LDCs satisfying (16), the elements $p_{i j}$, for $i=1,2, \ldots, 2 m-1$ and $j=i+1, i+2, \ldots, 2 m$, of the upper triangular matrix $\mathbf{P}$ in (10) are all zeros. For simplicity, we omit the proof in this paper.

\section{B. Simplified SD}

Note that $\mathbf{a}=\mathbf{y}-\mathbf{P} \tilde{\tilde{\mathbf{s}}}$. Thanks to the zeros in $\mathbf{P}, a_{i}$ (for $i=1, \ldots, 2 m)$ is a function of only $\tilde{\tilde{s}}_{i}$ and $\tilde{\tilde{s}}_{2 N}, \tilde{\tilde{s}}_{2 N-1}, \ldots, \tilde{\tilde{s}}_{2 m-1}$, rather than $\tilde{\tilde{s}}_{2 N}, \tilde{\tilde{s}}_{2 N-1}, \ldots, \tilde{\tilde{s}}_{i}$. As a result, $\tilde{\tilde{s}}_{i}$ (for $i=1, \ldots, 2 m$ ) appears only in the $i^{\text {th }}$ level. So to make a decision on $\tilde{\tilde{s}}_{i}$ (for $i=1, \ldots, 2 m)$, we do not need the knowledge of the $(i+1)^{\text {th }}$ level to the $2 m^{\text {th }}$ level. Furthermore, since $\tilde{\tilde{s}}_{i}$ (for $i=1, \ldots, 2 m$ ) does not affect any other level, to decide the value of $\tilde{\tilde{s}}_{i}$, we only need to choose the value of $\tilde{\tilde{s}}_{i}$ that minimizes $a_{i}$, rather than calculating the upper and lower bounds of $\tilde{\tilde{s}}_{i}$. Thus the decoding for these symbols is simply hard decoding:

$$
\tilde{\tilde{s}}_{i}=\left\lceil\left(y_{i}-\sum_{k=2 m+1}^{2 N} p_{i, k} \tilde{\tilde{s}}_{k}\right) / p_{i, i}\right\rfloor, \text { for } i=1, \ldots 2 m
$$

where $\lceil a\rfloor$ means the possible value of $\tilde{\tilde{s}}_{i}$ closest to $a$. It should be noted that, compared to the complexity of tree search, the complexity of hard decoding can be neglected and our proposed code structure can reduce the tree search by $2 m$ levels in SD without causing any degradation in BER performance.

\section{Simulation Results}

The advantages of the orthogonal structure for different modulations were studied in [15]. Here we investigate the LDCs for different numbers of transmit and receive antennas. Studies of this family of LDCs have been carried out using a $2 \times 4$ MIMO system transmitting eight QPSK symbols in a block length of 4 (i.e. $N_{t}=2, N_{r}=4, T=4, N=8$ ) and a $3 \times 3$ MIMO system transmitting ten QPSK symbols in a block length of 6 (i.e. $N_{t}=3, N_{r}=3, T=6, N=10$ ) over a blockfading channel. In the construction of these LDCs with orthogonal row structure, we first made the first two dispersion matrices to satisfy the orthogonal condition in (16), i.e. $m=2$. Then we used random search with the Rank \& Determinant criterion to obtain the optimal LDCs. For the LDCs without the orthogonal row structure, we simply use random search with the Rank \& Determinant criterion to obtain the optimal LDC. To assess the BER performances of these optimum LDCs, Monte Carlo simulation was used and the results are shown in Fig. 1. It can be seen that the BER performance of the optimum LDCs with and without the orthogonal row structure are about the same.

Here, the complexity of SD is measured by the number of nodes visited in the tree search process [2]. In the $2 \times 4 \mathrm{MIMO}$ systems transmitting eight QPSK symbols in a block length of 4 , the SD needs 16 levels in decoding. However, the simplified SD algorithm can reduce the decoding to a 12-level SD and 4 hard decisions, reducing $25 \%$ of the search levels. The complexity reduction is not linearly proportional to the number of search levels because the search process depends on many random factors, so it is difficult to estimate the actual complexity reduction. Monte Carlo simulation again was used to evaluate the complexities of the conventional SD and simplified SD for decoding the optimum LDCs with the orthogonal row structure. The results on the complexities for the $2 \times 4$ MIMO system transmitting eight QPSK symbols in a block length of 4 and the $3 \times 3$ system transmitting ten QPSK symbols in a block length of 6 are shown in Fig. 2 and Fig. 3 respectively. In the $2 \times 4$ system, the simplified SD reduces the complexity by $28-52 \%$; while in the $3 \times 3$ system, the simplified $\mathrm{SD}$ reduces the complexity by $28-49 \%$. 


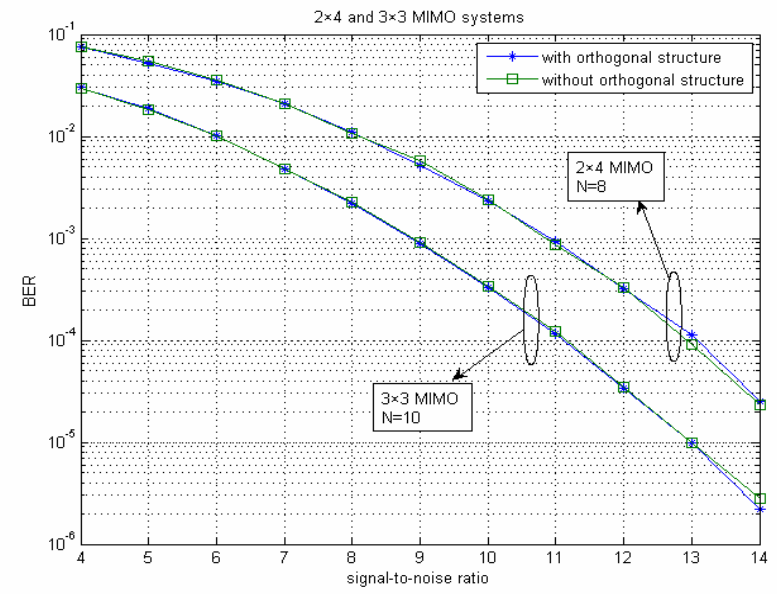

Figure 1. BERs of optimal LDCs in $2 \times 4$ and $3 \times 3$ MIMO systems.

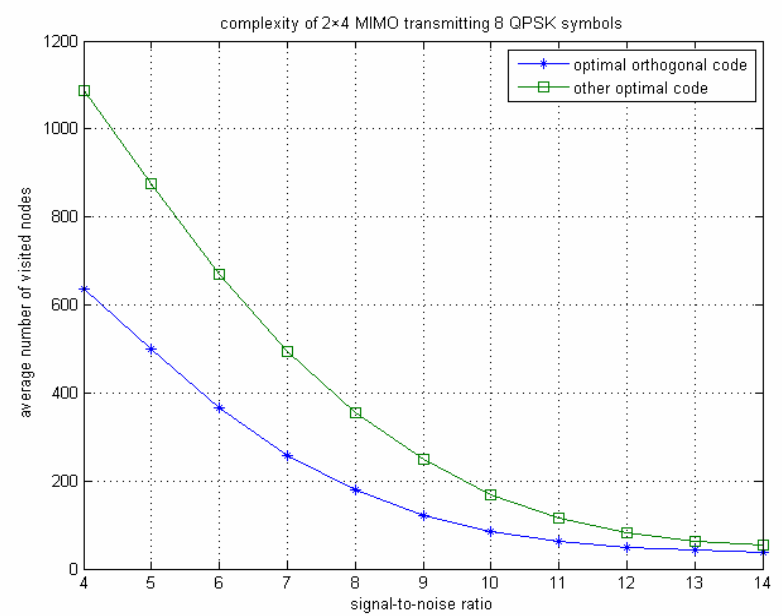

Figure 2. Complexities of conventional and simplified SDs in $2 \times 4 \mathrm{MIMO}$ system.

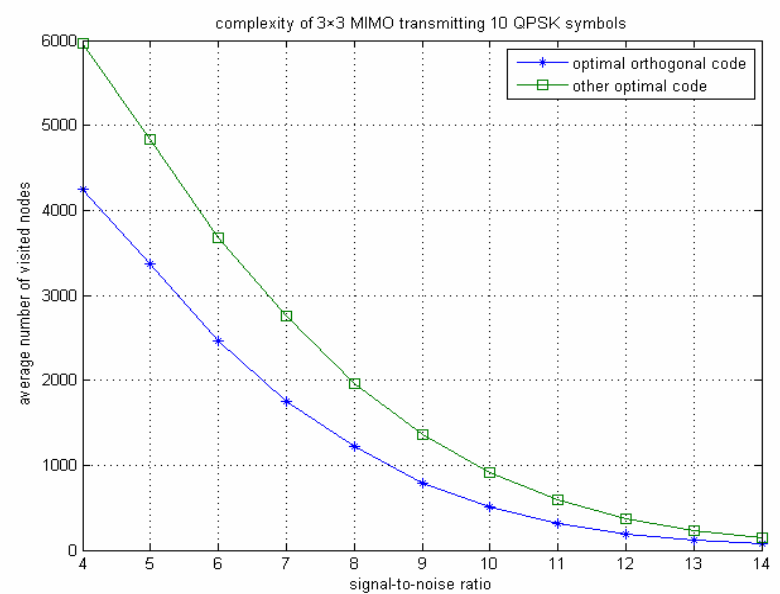

Figure 3. Complexities of conventional and simplified SDs in $3 \times 3 \mathrm{MIMO}$ system.

\section{CONCLUSIONS}

In this paper we have presented the design of a family of fast-decodable full diversity LDC with an orthogonal row structure and investigated these LDCs for different numbers of transmit and receive antennas. Monte Carlo computer simulations have shown that the optimal LDCs with and without the orthogonal row structure for different numbers of transmit and receive antennas have nearly identical BER performances. However, the complexity of SD for LDCs with orthogonal row structure can be significantly reduced by using a simplified SD algorithm. For a $2 \times 4$ MIMO system transmitting eight QPSK symbols in a block length of 4 and a $3 \times 3$ MIMO system transmitting ten QPSK symbols in a block length of 6 , the respective reductions are about $28-52 \%$ and 28 $49 \%$.

\section{REFERENCES}

[1] R. Heath and A. Paulraj, "Linear Dispersion Codes for MIMO Systems Based on Frame Theory', IEEE Trans. Sig. Proc., vol. 50, no. 10, pp. 2429-2441, October, 2002.

[2] V. Tarokh, N. Seshadri and A. R. Calderbank, "Space-Time Codes for High Data Rate Wireless Communication: Performance Criteria in the Presence of Channel Estimation Errors, Mobility, and Multiple Paths,', IEEE Trans. Commun., vol. 47, no. 2, pp. 199-207, February, 1999.

[3] G. D. Golden, G. J. Foschini, R. A. Valenzuela and P. W. Wolniansky, "Detection algorithm and initial laboratory results using the V-BLAST space-time communication architecture", Electronics Letters, vol. 35, no. 1, pp. 14-15, January, 1999.

[4] S. M. Alamouti, "A Simple Transmit Diversity Technique for Wireless Communications", IEEE Journal Select. Areas Commun., vol. 16, no. 8, pp. 1451-1458, October, 1998.

[5] V. Tarokh, H. Jafarkhani and A.R. Calderbank, "Space-time block codes from orthogonal designs," IEEE Trans. Inf. Theory, vol. 45, no. 5, pp. 1456-1467, July 1999.

[6] O. Tirkkonen and A. Hottinen, "Square-matrix embeddable space-time block codes for complex signal constellations", IEEE Trans. Inf. Theory, vol. 48, no. 2, pp. 384-395, February 2002.

[7] H. Jafarkhani, "A quasi-orthogonal space-time block code", IEEE Commun. Letters, vol. 49, no. 1, pp. 1-4, January, 2001.

[8] U. Fincke and M. Pohst, "Improved methods for calculating vectors of short length in lattice, including a complexity analysis", Math. Comput., vol. 44, no. 170, pp. 463-471, April, 1985.

[9] J. Jalden and B. Ottersten, "On the complexity of sphere decoding in digital communications", IEEE Trans. Sig. Proc., vol. 53, no. 4, pp. 1474-1484, April, 2005.

[10] E. Agrell, T. Eriksson, A. Vardy and K. Zeger, "Closest point search in lattices", IEEE Trans. Inf. Theory, vol. 48, no. 8, pp. 2201-2214, August 2002.

[11] C. P. Schnorr and M. Euchner, "Lattice basis reduction: Improved practical algorithms and solving subset sum problems," Math. Programming, vol. 66, pp. 181-191, 1994.

[12] J. Paredes, A.B. Gershman and M. G. Alkhanari, "A $2 \times 2$ space-time code with non-vanishing determinants and fast maximum likelihood decoding”, Proc. ICASSP., vol. 2, pp. 877-880, April, 2007.

[13] E. Biglieri, Y. Hong and E. Viterbo, "On fast decodable space-time block codes", IEEE Trans. Inf. Theory, Feb 2009.

[14] X.G. Dai, S. W. Cheung and T. I. Yuk, "A Fast-Decodable Code Structure for Linear Dispersion Codes”, IEEE Trans. on Wireless Commun. Aug, 2009.

[15] X.G. Dai, S. W. Cheung and T. I. Yuk., "A New Family of Linear Dispersion Code for Fast Sphere Decoding," Canadian Conference on Electrical and Computer Engineering 09, pp.314-317, May 2009. 